\title{
Key Technologies in Connected Autonomous Electrified Vehicles
}

\author{
Yugong Luo ${ }^{1}$, Wenbo $\mathrm{Chu}^{2}$ and Dongpu Cao ${ }^{3 *}$
}

The rapid development of technologies such as artificial intelligence, Internet of Things, big data, and information communication has promoted the development of connected autonomous electrified vehicles with functions such as complex environment perception, intelligent decision-making, collaborative control, and execution. The main objective of this special issue aims at bringing scholars to show their latest research results in environmental perception systems, autonomous driving decision-making systems, vertical and horizontal control systems, network communication systems, battery energy management systems, etc., and promote its application in the area of connected autonomous electrified vehicles in China. Eight papers have been selected in this special issue after rigorous review and they represent the latest research outcomes in this active area.

To achieve autonomous driving, several steps, including environment perception, path-planning, and dynamic control, need to be done. However, vehicles equipped with on-board sensors still have limitations in acquiring necessary environmental data for optimal driving decisions. The article "Cloud Control System Architectures, Technologies and Applications on Intelligent and Connected Vehicles: A Review" by Li et al. introduce Intelligent and connected vehicles (ICV) cloud control system (CCS) as a new concept as it is a potentially synthetic solution for high level automated driving to improve safety and optimize traffic flow in intelligent transportation. This paper systematically investigated the concept of cloud control platform from its architectures, core

\footnotetext{
*Correspondence: oscar_cao2016@163.com

${ }^{3}$ Department of Mechanical and Mechatronics Engineering, University of Waterloo, Waterloo, ON N2L 3G1, Canada

Full list of author information is available at the end of the article
}

technologies, design methodology, as well as application potentials on ICV. Based on the analysis, the challenges and suggestions on cloud control system development have been addressed.

As wireless communication advances, vehicle infrastructure integrated system approaches to intersection planning and decision making have received increased attention. "Planning and Decision-making for Connected Autonomous Vehicles at Road Intersections: A Review" by Cao et al. primarily overview recent studies on the planning and decision-making technologies at intersections, which allow vehicles to pass the intersection safely and efficiently. This paper presents the general planning and decision-making approaches: graph, predictionbased, optimization, machine learning, end-to-end, reinforcement learning, and partially observable Markov methods. The paper also summaries the evolving planning decision-making methods based on vehicle infrastructure cooperative technologies.

In "Energy-Optimal Braking Control Using a DoubleLayer Scheme for Trajectory Planning and Tracking of Connected Electric Vehicles", Yin et al. present an energyoptimal braking strategy (EOBS) to improve the energy efficiency of EVs with the consideration of shared braking intention. First, a double-layer control scheme is formulated. Then, this paper conducted several simulations by jointing MATLAB and CarSim, the results demonstrated the proposed EOBS achieves prominent regeneration energy improvement than the regular constant deceleration braking strategy. Finally, the energy-optimal braking mechanism of EVs is investigated based on the analysis of braking deceleration, battery charging power, and motor efficiency, which could be a guide to real-time control.

The work by Guo et al. entitled "Neural-Fuzzy-Based Adaptive Sliding Mode Automatic Steering Control of 
Vision-based Unmanned Electric Vehicles" presents a novel neural-fuzzy-based adaptive sliding mode automatic steering control strategy to improve the driving performance of vision-based unmanned electric vehicles with time-varying and uncertain parameters. The kinematic and dynamic models which accurately express the steering behaviors of vehicles are constructed. Then, a neural-fuzzy-based adaptive sliding mode automatic steering controller is proposed to supervise the lateral dynamic behavior of unmanned electric vehicles. The results illustrate the presented control scheme has the excellent properties in error convergence and robustness.

"Surrounding Objects Detection and Tracking for Autonomous Driving Using LiDAR and Radar Fusion" by Cai et al. present an effective method for high-precision detection and tracking of surrounding targets of autonomous vehicles. By employing the Unscented Kalman Filter, Radar and LiDAR information is effectively fused to achieve high-precision detection of the position and speed information of targets around the autonomous vehicle. Finally, the real vehicle test under various driving environment scenarios is carried out. The experimental results show that the proposed sensor fusion method can effectively detect and track the vehicle peripheral targets with high accuracy.

In "Rollover Prevention and Motion Planning for an Intelligent Heavy Truck", Wang et al. propose a motion planning strategy with autonomous anti rollover ability for an intelligent heavy truck to improve rollover stability. This paper built the rollover dynamics model for the intelligent heavy truck. Then, the optimal path is obtained to meet the requirements that the intelligent heavy truck can avoid obstacles and drive stably without rollover. In addition, three typical scenarios are designed to numerically simulate the dynamic performance of the intelligent heavy truck. The results show that the proposed motion planning strategy can avoid collisions and improve vehicle rollover stability effectively even under the worst driving scenarios.

The research by Yin et al. entitled "Adaptive Multimodal Fusion Instance Segmentation for CAEVs in Complex Conditions: Dataset, Framework and Verifications" introduce an end-to-end sharpening mixture of experts (SMoE) fusion framework to improve the robustness and accuracy of the perception systems for CAEVs in complex illumination and weather conditions. In this paper, the Complex KITTI dataset is introduced, and this dataset is fine annotated in instance-level with the proposed semiautomatic annotation method. The SMoE fusion approach is devised to adaptively learn the robust kernels from complementary modalities. The experimental results show that the proposed SMoE framework yield significant improvements over the other fusion techniques in adverse environmental conditions.

Finally, "ML-ANet: A Transfer Learning Approach Using Adaptation Network for Multi-label Image Classification in Autonomous Driving" by Li et al. propose a new multi-label adaptation network (ML-ANet) based on multiple kernel variants with maximum mean discrepancies to reduce the discrepancy between the source and target domains. This paper also conducted adverse weather and cross-camera adaptation examinations to verify the effectiveness of the proposed ML-ANet. The results show that ML-ANet achieves higher accuracies than the compared state-of-the-art methods for multilabel image classification in both the adverse weather adaptation and cross-camera adaptation experiments.

From the papers published in this Special Issue, we hope they will benefit the development of connected autonomous electrified vehicles research community.

\section{Acknowledgements \\ Not applicable.}

\section{Authors' Information}

Yugong Luo, born in 1974, is currently a professor at State Key Laboratory of Automotive Safety and Energy, Tsinghua University, China. His research interests include Electric vehicles, vehicle dynamics and control.

Wenbo Chu, born in 1986, is currently a Research Fellow at China Intelligent and Connected Vehicles (Beijing) Research Institute Co., Ltd, China, which is also the National Innovation Center of Intelligent and Connected Vehicles. He received his B.S. degree majored in Automotive Engineering from Tsinghua University, China, in 2008, and his M.S. degree majored in Automotive Engineering from RWTH-Aachen, German and Ph.D. degree majored in Mechanical Engineering from Tsinghua University, China, in 2014.

Dongpu Cao, born in 1978, is currently a professor at Department of Mechanical and Mechatronics Engineering, University of Waterloo, Canada. He received his PhD degree from Concordia University, Canada, in 2008. His research interests include vehicle dynamics, control, and intelligence.

\section{Authors' Contributions}

All authors read and approved the final manuscript.

\section{Competing interests}

The authors declare that they have no competing interests.

\section{Author Details}

${ }^{1}$ State Key Laboratory of Automotive Safety and Energy, Tsinghua University, Beijing 100084, China. ${ }^{2}$ China Intelligent and Connected Vehicles (Beijing) Research Institute Co., Ltd, Beijing 100176, China. ${ }^{3}$ Department of Mechanical and Mechatronics Engineering, University of Waterloo, Waterloo, ON N2L 3G1, Canada.

Received: 8 November 2021 Accepted: 12 November 2021

Published online: 20 December 2021 